\title{
Gelöscht und doch nicht gelöscht
}

\author{
Prüfung von Polizeidatenbeständen
}

\author{
Ulrike Müller, Alfons Schranz
}

Das Verfahren der Speicherung und Löschung in POLAS-HE, dem Hessischen polizeilichen Auskunftssystem, stützt sich auf die $\S \S 20$ Abs. 4 und 27 Abs. 4 des Hessischen Gesetzes über die öffentliche Sicherheit und Ordnung (HSOG) und die dazu vom Hessischen Ministerium des Innern und für Sport erlassene Prüffristenverordnung (PrüffristVO). Danach erhält jeder in POLAS-HE gespeicherte Datensatz zwingend nach Abschluss der Ermittlungen ein so genanntes Aussonderungsprüfdatum. Jeden Monat wird eine Prüfliste mit den Fällen erstellt, bei denen in vier Monaten dieses Datum verstreicht und die Person während der Dauer der Datenspeicherung nicht erneut in den Verdacht geraten ist, Straftaten begangen zu haben. Diese Liste wird den zuständigen Polizeipräsidien zur Verfügung gestellt, um in begründeten Einzelfällen die Frist zu verlängern. Liegen keine Gründe für die Verlängerung vor, werden die Datensätze nach vier Monaten automatisch gelöscht. Danach werden den Akten führenden Stellen Listen der gelöschten Datensätze übersandt, damit diese die dazugehörigen Kriminalakten und evtl. vorhandene erkennungsdienstliche Unterlagen - Lichtbilder und Fingerabdrücke aussondern und vernichten. Durch die Verordnung und das Verfahren ist grundsätzlich sichergestellt, dass die Fristen eingehalten werden.

Nachdem mehrere Fälle bekannt geworden sind, in denen das Datum verstrichen, kein Grund für eine Fortdauer der Datenspeicherung zu erkennen war, die Daten aber nicht gelöscht waren, hat der Hessische Datenschutzbeauftragte das Hessische Präsidium für Technik, Logistik und Verwaltung (PTLV) gebeten, die Datenbank POLAS-HE für Zwecke der Datenschutzkontrolle auszuwerten. Es sollten Datensätze aufgelistet werden, deren Prüfdatum im Juli 2005 zur Löschung führen sollte. Da in den auffällig gewordenen Einzelfällen die Betroffenen immer erkennungsdienstlich behandelt worden waren, sollte die Auswertung auch nur solche Fälle enthalten. Einige Wochen nach Ablauf des Datums wurde die Liste der Datensätze mit den verstrichenen Prüfdaten im Polizeicomputer abgefragt. Das Ergebnis überraschte: Keiner der abgefragten Datensätze war gelöscht.

Eine Analyse ergab folgendes Bild: $\mathrm{Zu}$ etwa einem Drittel gab es zu den Personen zusätzliche Datenspeicherungen von Polizeibehörden anderer Bundesländer oder des Bundeskriminalamtes mit späteren Aussonderungsprüfdaten. In diesen Fällen hatten technische Vorkehrungen zu Recht bewirkt, dass die Daten nicht gelöscht waren.

$\mathrm{Zu}$ einem weiteren Teil gab es zusätzliche Datenspeicherungen die nicht von anderen Polizeivollzugsbehörden, sondern von Ausländerbehörden über ausländerrechtliche Entscheidungen stammten. Im Gegensatz $\mathrm{zu}$ Entscheidungen anderer Polizeibehörden gibt es bei Entscheidungen von Ausländerbehörden keinen Rechtsgrund, der die Vernichtung einer ansonsten löschungsreifen polizeilichen Information hindert. Es hätten deshalb nur noch die ausländerrechtlichen Entscheidungen, nicht aber die kriminalpolizeilichen Daten gespeichert sein dürfen.

Bei einem weiteren Anteil waren die Datensätze als „überregional relevant“ gekennzeichnet. Diese Kennzeichnung bewirkt, dass der jeweilige Datensatz auch außerhessischen Polizeibehörden zur Verfügung steht. Damit hat die Landespolizei die Verfügungshoheit über den Datensatz aufgegeben. Der Anstoß zur Datenlöschung muss nun vom Bundeskriminalamt erfolgen. $\mathrm{Ob}$ und wann dieser Anstoß erfolgt, war aber dem Landeskriminalamt nicht bekannt. Offensichtlich hält sich das BKA nicht an das von der Landespolizei verfügte Datum.

Bei weiteren Fällen ohne ,überregionale Relevanz" war im polizeilichen Auskunftssystem POLAS-HE tatsächlich kein Datensatz mehr vorhanden. Trotzdem zeigte das
Auskunftssystem, mit dem gleichzeitig das bundesweite Informationssystem abgefragt wird, immer dann einen „Treffer“, wenn die Person erkennungsdienstlich behandelt worden war. Das erklärt sich daraus, dass das Bundeskriminalamt ein Mehrexemplar jeder erkennungsdienstlichen Behandlung erhält. Dies wiederum führt zu einer Datenspeicherung im bundesweiten polizeilichen Informationssystem, die nur auf Veranlassung des Bundeskriminalamtes gelöscht werden kann. Auch in diesen Fällen konnte das Landeskriminalamt dem Datenschutzbeauftragten nicht darlegen, wann das Bundeskriminalamt die Löschung veranlasst. Offensichtlich sind die Fristen nicht identisch.

Bei einem letzten Teil von Vorgängen, bei denen das Aussonderungsprüfdatum verstrichen war, war die versäumte Löschung nicht nachvollziehbar. Auch nach Einschätzung der Polizei war in diesen Fällen eindeutig kein Grund für die unterbliebene Löschung ersichtlich.

\section{Fazit}

Theoretisch gibt es ein klares, datenschutzrechtlich akzeptables Verfahren, wann Daten aus dem polizeilichen Informationssystem POLAS-HE gelöscht werden Es genügt datenschutzrechtlichen Anforderungen. Allein: Es funktioniert nicht. Das Landespolizeipräsidium wurde auf die Feststellungen hingewiesen und um dringliche Behebung des Missstandes gebeten.

\section{Ulrike Müller \\ Regierungsdirektorin beim Hessischen Datenschutzbeauftragten \\ Alfons Schranz \\ Regierungsoberrat beim Hessischen Daten- schutzbeauftragten \\ poststelle@datenschutz.hessen.de}

